Revista de
Economida
Contemporâned

\title{
A ECONOMIA NÃO OBSERVADA NO BRASIL: UM ESTUDO BASEADO NA METODOLOGIA DO SISTEMA DE CONTAS NACIONAIS
}

\author{
João Hallak Neto ${ }^{a}$ \\ Roberto Olinto Ramos ${ }^{b}$ \\ ${ }^{a}$ Analista do Instituto Brasileiro de Geografia e Estatística (IBGE). \\ ${ }^{\mathrm{b}}$ Chefe da Coordenação de Contas Nacionais do IBGE.
}

Artigo recebido em 13/09/2013 e aceito em 17/01/2014.

RESUMO: As considerações que envolvem as atividades produtivas não captadas regularmente pelas estatísticas oficiais, a "economia não observada" (ENO), é tema relevante para a economia brasileira, associado diretamente ao Sistema de Contas Nacionais (SCN). Este artigo busca trazer à baila questões conceituais, metodológicas e resultados para a economia brasileira de forma a complementar o SCN correntemente divulgado no país. A ENO no Brasil como proporção do PIB decresceu de 15,8\% para 11,6\%, entre 2000 e 2009, resultado influenciado pela redução do ainda significativo peso da produção familiar e informal na economia do País. Fatores como o crescimento econômico, sobretudo no período 2004-2008, e efeitos de medidas específicas como redução e simplificação de impostos, expansão do crédito para microempreendedores e o aumento da fiscalização trabalhista, foram identificados como relevantes para esta redução.

PALAVRAS-CHAVE: Economia não observada; economia informal; economia subterrânea; sistema de contas nacionais.

CLASSIFICAÇÃO JEL: E26.

Correspondência para João Hallak Neto.

E-mail: joao.hallak@ibge.gov.br. 


\title{
THE BRAZILIAN NON-OBSERVED ECONOMY: A STUDY ESTABLISHED ON THE SYSTEM OF NATIONAL ACCOUNTS METHODOLOGY
}

\begin{abstract}
The considerations about productive activities regularly not captured by official statistics, the "non-observed economy" (NOE), is a relevant issue for the Brazilian economy, linked directly to the System of National Accounts (SNA). This article seeks to bring up conceptual and methodological questions, as well as results for the Brazilian economy to complement the SCN commonly reported in the country. The ENO in Brazil as a proportion of GDP declined from $15.8 \%$ to $11.6 \%$ between 2000 and 2009, a result influenced by reducing the still significant weight of household production and the informal economy Factors such as economic growth, especially in the period 2004-2008, and effects of specific policies such as reducing and simplifying taxes, credit expansion for micro entrepreneurs and increased labor inspection were identified as relevant to this reduction.
\end{abstract}

KEYWORDS: Non-observed economy; informal economy; underground economy; system of national accounts. 


\section{INTRODUÇÃO}

A cobertura completa da produção econômica em um país é extremamente importante para assegurar uma boa qualidade das informações prestadas pelo Sistema de Contas Nacionais (SCN) e, consequentemente, para que este cumpra sua função de representar quantitativamente a atividade econômica de uma determinada realidade histórica, configurando-se em um dos principais instrumentos para o planejamento econômico. Para garantir esta cobertura completa, ou exaustiva - termo utilizado na literatura internacional, um trabalho significativo é requerido em razão das dificuldades de se contabilizar certos tipos de atividades que não podem ser captadas diretamente pelo sistema regular de estatística. A este grupo de atividades convencionou-se chamar de Economia Não Observada (ENO).

A ENO é composta de atividades produtivas realizadas pelas famílias informalmente ou para o uso próprio, atividades que são deliberadamente subdeclaradas pelos produtores, atividades ilegais, e ainda, as estatisticamente subcobertas. O propósito de um sistema estatístico nacional é assegurar, tanto quanto possível, que as atividades produtivas, observadas ou não, sejam apropriadamente contabilizadas e incluídas nos resultados das contas nacionais. Assim, a economia não observada não deve ser confundida com a economia não mensurada.

A questão da ENO, de desenvolvimento recente, tem recebido crescente atenção das divisões de estatística de diversos organismos internacionais, tais como a Organização para a Cooperação e Desenvolvimento Econômico (em inglês, Organisation for Economic Co-operation and Development / OECD), o Fundo Monetário Internacional (FMI) e a Comissão de Estatísticas Econômicas para a União Europeia (em inglês, United Nations Economic Commission for Europe / UNECE). Estas instituições desenvolveram uma série de ações no sentido de promover uma harmonização em torno de seu conceito e de apresentar métodos padronizados de sua mensuração para serem aplicados pelos diversos países ${ }^{1}$.

No Brasil, assim como nos demais países em desenvolvimento, as atividades produtivas familiares e informais possuem relevância para a geração da renda, além de se constituírem importante fonte de emprego para um grande número de trabalhadores. Entretanto, usualmente, seus resultados são inferiores aos que indicam algumas das projeções calculadas por modelos econométricos que não consideram o marco teórico do SCN. O equívoco sobre esta questão é ainda mais grave quando se infere que os cálculos das contas nacionais não incluem este tipo de produção. Esta é a origem de

1 Alguns exemplos de trabalhos com recomendações padronizadas são UNECE (1993, 2003 e 2008) e OECD (2002). 
afirmações implausíveis como a que o Brasil possui, em termos de renda, uma "Argentina escondida”, não contabilizada em uma de suas principais referências estatísticas para estudos econômicos.

Este artigo tem como objetivo apresentar as principais técnicas recomendadas para a busca da exaustividade da cobertura econômica e, a partir do uso delas no SCN brasileiro, propor uma estimativa da ENO para o país. Este objetivo tornou-se viável após a introdução dos avanços incorporados ao sistema estatístico brasileiro na base de dados das contas nacionais, que ocorreram a partir da publicação da nova série de dados do SCN, em março de $2007^{2}$. A série histórica com os resultados disponíveis inicia-se em 2000, ano de referência da nova série, e termina em 2009, último ano de publicação do SCN do Brasil na versão detalhada pelos setores institucionais.

A relevância de se tratar deste tema, sob a perspectiva da economia brasileira, deve-se também ao fato de que parte significativa da ENO está ligada à produção das unidades familiares. Esta, embora decrescente em termos proporcionais nos anos finais da série, possui ainda um peso relevante na geração do valor adicionado no país ${ }^{3}$. Estas atividades constituem-se uma importante fonte de trabalho e de rendimentos para um grande número de pessoas no Brasil e nas demais economias dos países em desenvolvimento.

Este artigo encontra-se estruturado em mais quatro itens além desta introdução. $\mathrm{O}$ segundo item apresenta o conceito de ENO, sua articulação com a fronteira da produção do SCN e seus componentes. No item 3 são destacados os principais métodos recomendados internacionalmente para a estimação da atividade não observada. $\mathrm{O}$ item 4 realiza uma estimativa para o caso brasileiro, onde se constata a redução do peso da ENO em virtude da queda de participação da produção familiar ao longo dos anos 2000. As considerações finais constam do último item.

\section{A ECONOMIA NÃO OBSERVADA}

O sistema estatístico nacional é composto por todas as organizações e unidades de um país que conjuntamente coletam, processam e disseminam as estatísticas oficiais ${ }^{4}$. As distintas instituições fornecem dados econômicos básicos de diversas fontes, como

2 O relatório metodológico (IBGE, 2008b) apresenta as modificações introduzidas na nova série do SCN do Brasil.

3 Em 2000, a contribuição do setor Famílias para a geração de valor adicionado foi de 27,2\%. Em 2009, este valor recuou para 20,0\% (IBGE, 2011).

4 A principal instituição deste conjunto é o instituto oficial de estatística. No caso do Brasil cabe ao IBGE este papel. 
pesquisas econômicas ou domiciliares, censos populacionais, registros administrativos, registros fiscais, balanços contábeis, entre outros. Esta infraestrutura estatística e os procedimentos de pesquisa que coletam e processam estes dados são denominados "programa de compilação de dados básicos"5. Posteriormente estas informações são fornecidas à área de contas nacionais para serem apropriadamente transformadas nos conceitos do SCN e em seguida utilizadas em seus cálculos ${ }^{6}$.

Embora em geral bastante numerosas e de diversas naturezas (social, econômica ou administrativa) e variando também de acordo com a unidade de investigação (empresa, unidade local, estabelecimento ou domicílio), as fontes de dados regulares que alimentam o sistema estatístico nacional estão sujeitas, em maior ou menor grau, ao que ficou denominado na literatura internacional como estatística subterrânea, ou seja, às deficiências no programa de compilação de dados básicos ${ }^{7}$, constituindo-se nas razões para a existência da ENO nos SCN dos países. Estas deficiências estão associadas a três tipos de natureza:

i) A subcobertura de unidades de interesse - quando unidades produtivas, ou partes delas, não estão incluídas no programa de compilação de dados básicos;

ii) A não resposta por parte das unidades informantes - as unidades estão representadas no universo da pesquisa, mas não há dados coletados para elas e a imputação de resultados para as observações ausentes não é adequadamente realizada;

iii) A subdeclaração por parte das unidades informantes - os dados são obtidos a partir das unidades produtivas, mas a informação foi ocultada, ou declarada parcialmente, de maneira deliberada pelo entrevistado; ou ainda, os dados recebidos foram corretos, mas registrados ou ponderados equivocadamente pelo órgão compilador.

Tais deficiências, passíveis de ocorrerem em qualquer sistema nacional de estatística, mostram a necessidade de melhorias contínuas nos programas de compilação de dados básicos, que, entre outras vantagens, objetivam reduzir ocorrência da ENO, garantindo a inclusão das atividades produtivas na maior extensão possível.

5 Do inglês, basic data collection programme (OECD, 2002).

6 Sobre o sistema estatístico nacional e as diversas fontes que formam as estatísticas econômicas integradas, bem como a centralidade do SCN como elemento organizador deste processo ver UNECE (2012).

7 Em inglês, deficiencies in the basic data collection programme (statistical underground) (Eurostat, 2010, p. 99). 
Desde os anos 1990, este tema tem recebido destaque nas comissões de estatística ligadas às contas nacionais. Em 1993, a UNECE divulgou um trabalho pioneiro (UNECE, 1993) em que tratou da contribuição de nove países sobre práticas de estimação de atividades da economia informal e subdeclarada para as contas nacionais. Este debate prosseguiu e um segundo levantamento foi realizado em 2001 pela mesma entidade, desta vez com 29 países membros (UNECE, 2003). Tal publicação contribuiu para a elaboração do Manual da OECD para a Economia Não Observada (OECD, 2002), que teve como principal objetivo prover um guia padronizado com a convergência de opinião entre estatísticos e especialistas no tema sobre o que se constituem as melhores práticas para a completa estimação do PIB considerando as atividades não observadas coerentemente com o enfoque das contas nacionais.

Após a difusão de parte do conhecimento registrado no Manual da OECD, a UNECE realizou, em 2008, um terceiro levantamento sobre as práticas de 43 países em relação ao tratamento da ENO (UNECE, 2008). Neste trabalho, os objetivos foram apresentar um inventário das práticas correntes de estimação da ENO nos distintos países, prover uma plataforma para a comparação das diferentes abordagens utilizadas e apresentar uma referência para os países que desejam ampliar seus esforços para a estimação exaustiva do PIB, levando em conta as atividades observadas e não observadas em seu cálculo (UNECE, 2008, p. 1).

Este investimento contínuo na investigação e na disseminação do conhecimento em relação ao tratamento da ENO endossa a necessidade de se reconhecer a existência deste fenômeno nas economias nacionais. Embora não declarada, a ENO deve ser mensurada e incorporada ao SCN com a finalidade de agregar qualidade e utilidade às contas nacionais em geral, e a seus principais agregados, como o PIB e a renda nacional, em particular. Ademais, a relevância de se estudar o tema está também no fato deste se constituir uma fonte de oportunidades de trabalho e renda para um grande número de pessoas - conforme denota o alto peso da economia familiar, particularmente a informal em um conjunto de países, sobretudo nas economias em desenvolvimento.

A busca pela completa mensuração da renda nacional é de extrema importância, ainda mais quando consideramos as importantes decisões que são derivadas deste indicador. Uma subavaliação da renda nacional implica em dificuldades para usuários e também para os responsáveis pela elaboração das contas nacionais. Para estes, a avaliação equivocada causa desequilíbrios internos na consistência do sistema, uma vez que algumas transações econômicas podem estar sendo mensuradas enquanto outras não.

Para os usuários do SCN, a deficiência na cobertura econômica causa problemas não só em termos de valor - um PIB subestimado, por exemplo; mas também de tendência, que reflete em imprecisas taxas de crescimento da economia. Isto ocorre porque o segmento não observado usualmente apresenta variação distinta, quando não inversa, 
do segmento observado. Por exemplo, é razoável supor que a produção realizada pelas Famílias tenha uma variação inversa à evolução do restante da economia. Em períodos de crescimento, a produção informal e de bens para o próprio uso tenderiam a aumentar menos rapidamente que a produção formal, pois a força de trabalho encontra melhores oportunidades de emprego nas unidades formais da economia, que apresentam crescimento. Tal fenômeno ocorreu no Brasil após o ciclo de crescimento econômico iniciado em 2004 que tornou o perfil da economia nacional mais formalizado nos anos seguintes. Por sua vez, na década de 1990, em que se registrou um baixo crescimento do PIB e uma maior taxa de desemprego, o setor informal ganhou participação na economia brasileira.

Além dos efeitos do dinamismo econômico, as alterações no arcabouço legal/institucional vigente no país também devem implicar em consequencias sobre os resultados da ENO. Por exemplo, uma alteração na legislação tributária ou nas condições de acesso ao crédito para as microempresas deverá exercer um impacto significativo na inclusão das unidades produtivas no setor formal. Assim, é seguro afirmar que os resultados, a extensão e a estrutura da parte não observada da economia variem consideravelmente entre os países e sejam dependentes, não só de aspectos como o alcance do sistema nacional de estatística, mas também, do estágio de desenvolvimento da economia, da legislação vigente e da organização da sociedade.

No contexto internacional é natural que a ENO apresente maior peso nos países em desenvolvimento em relação aos países mais desenvolvidos por causa da maior informalidade e da menor fiscalização relativas nos primeiros. A cobertura do sistema nacional de estatística, que tende a ser maior nos países mais desenvolvidos, também explica a menor parcela de ENO verificada nestes.

\section{A ARTICULAÇÃO ENTRE A FRONTEIRA DE PRODUÇÃO E A ENO}

Um conceito fundamental para a elaboração das contas nacionais é o de produção. As regras que determinam o que deve ser incluído ou não como produção definem a fronteira de produção do SCN. Esta, por sua vez, determina o escopo da maioria das transações nas contas nacionais, a começar pelo que deve ser considerado como produto. Como para o SCN, somente são reconhecidos os usos de bens e serviços produzidos, a fronteira da produção delimita também o âmbito do consumo intermediário, e, portanto, do valor adicionado - que é a diferença entre a produção e o consumo intermediário. Pela mesma razão, a fronteira da produção define também o que deve ser incluído como o consumo das famílias e os outros usos finais. Ademais, como SCN reconhece apenas as rendas geradas no processo produtivo, a fronteira da produção também determina o que deve ser incluído como rendimento, e consequentemente 
como poupança, a diferença entre a renda disponível e o consumo final, e a capacidade ou a necessidade de financiamento, o saldo das contas correntes e de capital no SCN.

Dessa forma, para o entendimento da ENO parte-se do pressuposto de que toda a produção de bens e serviços que esteja dentro da fronteira da produção deve ser considerada para os cálculos das variáveis das contas nacionais. Esta tem seus limites definidos, segundo o SNA-2008, como toda a produção destinada ao mercado, quer à venda ou à permuta. Inclui ainda os bens e serviços fornecidos gratuitamente às famílias ou à comunidade pelos serviços da administração pública ou pelas instituições sem fins de lucro a serviço das famílias, bem como algumas atividades realizadas pelas famílias para o próprio uso (UN, 2009, par. 1.40, p. 6).

De tempos em tempos as definições conceituais para a elaboração dos SCN sofrem modificações referendadas no âmbito de organismos internacionais por representantes dos diversos países em comitês estatísticos ou de contas nacionais. Estas alterações representam, na medida do possível, as mudanças nas economias e nas sociedades e são também influenciadas pelas ideologias que assumem posições hegemônicas em determinados períodos históricos, passando a incorporar as metodologias do SCN. Para mencionar o período mais recente, a definição do escopo da fronteira de produção registrou, com a publicação do SNA-1993, uma pequena alteração em relação à versão de 1968 (UN, 1993, p. 653), ampliando o limite de produção para as atividades de produção familiar, que incluiu a produção dos serviços, exceto os considerados para o uso final, que não os serviços de proprietários residentes (aluguel imputado) e aqueles produzidos pelo emprego de pessoal doméstico remunerado.

Em que pese este aumento dos limites da produção em relação à versão de 1968, a produção de serviços pelas famílias para consumo final próprio, tais como preparo de refeições, educação e cuidados com crianças, limpeza, reparação e manutenção dos bens duráveis de consumo e da habitação, permaneceu fora do SCN desde a sua primeira edição até hoje. A explicação para a exclusão dos serviços domésticos e pessoais não remunerados é que estas modalidades de produção dentro das famílias são atividades autônomas, de difícil atribuição de valor e de impacto muito restrito na economia. Sendo assim, no quadro central do sistema, nenhum valor é registrado para estes serviços. Diferentemente da produção de bens para o próprio consumo, não há preços de mercado que poderiam ser satisfatoriamente utilizados para valorar tais serviços. Ademais haveria uma repercussão limitada no resto da economia que traria mais desvantagens do que benefícios ao utilizar este conjunto de dados para fins analíticos ou de proposições políticas. Segundo o SNA-1993:

The 1993 SNA explains that in the central framework no values are recorded for unpaid domestic or personal services produced within households because the 
production of such services within households is a self-contained activity with limited repercussions on the rest of the economy, there are typically no prices that can be satisfactorily used to value such services, and the estimated values would not be equivalent to monetary values for analytic or policy purposes. (UN, 1993, p. 653)

Outro argumento que reforça a exclusão dos serviços pessoais e domésticos não remunerados é que, de acordo com diretivas da OIT, as pessoas economicamente ativas são aquelas que estão envolvidas em atividades produtivas, tal como estas são entendidas pelo SCN. Se os limites desta produção fossem alargados de forma a incluir a produção dos serviços pessoais e domésticos pelos membros das famílias para consumo próprio, o desemprego seria, por definição, virtualmente inexistente, tornando o uso deste conceito sem relevância para a maioria dos usuários (UN, 1993, p. 6):

The location of the production boundary in the System is a compromise, but a deliberate one that takes account of the needs of most users. In this context it may be noted that in labour force statistics economically active persons are defined as those engaged in productive activities as defined in the SNA. If the production boundary were extended to include the production of personal and domestic services by members of households for their own final consumption, all persons engaged in such activities would become self-employed, making unemployment virtually impossible by definition. This illustrates the need to confine the production boundary in the SNA and other related statistical systems to market activities or fairly close substitutes for market activities. (UN, 1993, p. 6, grifo dos autores)

Entretanto, o SNA-93 admite e até sugere que em contas satélites um conceito alternativo do produto interno bruto (PIB) possa ser elaborado tendo como base um limite de produção ampliado, que inclua as estimativas para a produção doméstica de serviços para uso próprio ${ }^{8}$.

A Figura 1 mostra um fluxograma que por meio de quatro perguntas diretas (sim ou não) e alguns exemplos esclarece se as atividades desempenhadas estão ou não presentes na fronteira da produção do SCN, sob os critérios definidos no SNA-1993 e mantidos na revisão do manual, o SNA-2008. Como mostrado no item 4, as atividades que formam a ENO estão consideradas na fronteira de produção do sistema.

\footnotetext{
8 Um estudo neste sentido envolvendo a economia brasileira é Melo et al. (2005).
} 
Figura 1 - Fronteira de produção segundo o SCN

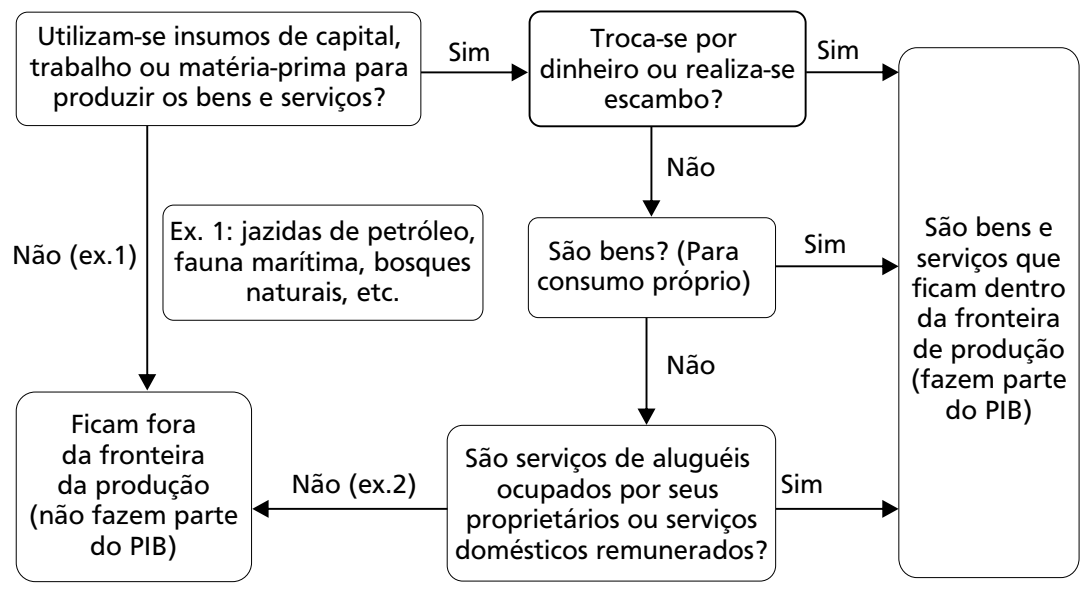

Ex. 2: cuidado filhos, alimentos, roupas, reparos, feito pela própria família

Embora tenha se mantido praticamente a mesma desde o SNA-1993, a definição de fronteira de produção sofreu algumas pequenas modificações na versão de 2008 do manual. Tais modificações dizem respeito a itens pontuais, como em relação à mudança de classificação da atividade de Pesquisa e desenvolvimento (P\&D), que deixou de ser considerada auxiliar e passou à principal, ao refinamento no método de cálculo dos Serviços de Intermediação Financeira Indiretamente Medidos (SIFIM) e, à definição mais clara da produção dos bancos centrais. Ainda em relação às atividades financeiras, houve também modificações no âmbito do registro da produção de serviços de seguros e da atividade de resseguro ${ }^{9}$.

\section{A ENO E SEUS COMPONENTES}

Conforme explicado no item 3, todo e qualquer tipo de atividade que esteja considerada dentro da fronteira da produção, deve ter sua produção contabilizada no SCN.

9 O anexo III do SNA-2008 (UN, 2009, p. 581-602) é dedicado às alterações entre uma versão e outra, não só em relação à fronteira de produção, mas também no que concerne a outros tópicos. 
Assim como as atividades que integram a economia observada, as atividades que fazem parte da ENO estão também contempladas na fronteira de produção e, por isso, devem ser incorporadas ao sistema. A Figura 2 ilustra essa ideia.

\section{Figura 2 - Espaço da fronteira da produção total da Economia - Economia Observada e ENO}

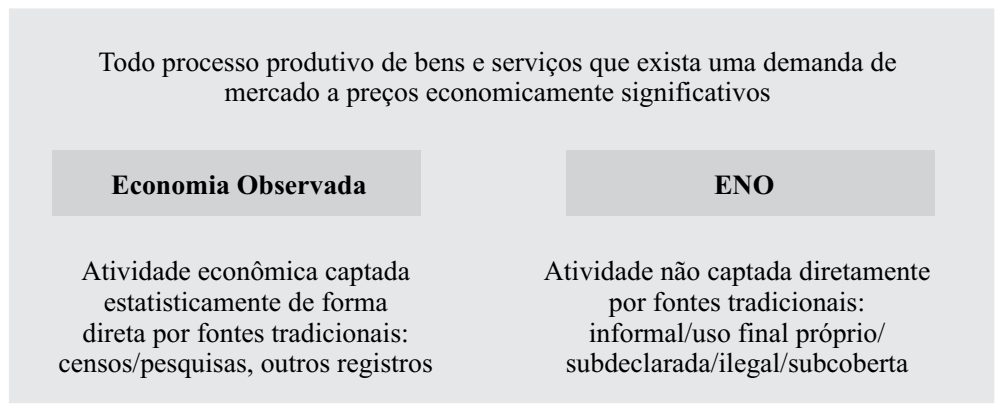

Fonte: Elaboração própria a partir das definições de OECD (2002).

As cinco categorias que potencialmente integram a ENO - ilustradas na Figura 2 - foram apresentadas originalmente no Manual de ENO (OECD, 2002, p. 13-14) e posteriormente nas outras publicações já mencionadas que tratam do tema. A descrição de cada uma delas é a seguinte:

\section{A) PRODUÇÃO DO SETOR INFORMAL}

Consiste na atividade de um conjunto de unidades produtivas não agrícolas, que se caracterizam por um baixo nível de organização e não possuem uma clara divisão entre trabalho e capital enquanto fatores de produção e cuja produção é destinada prioritariamente ao mercado ${ }^{10}$. Tal produção situa-se no setor institucional famílias, segmento aonde são contabilizados os empreendimentos produtivos que não são registrados formalmente. Em geral, a produção informal possui maior peso nas atividades econômicas de serviços, comércio e construção civil.

\footnotetext{
${ }^{10}$ Esta definição foi apresentada originalmente na 15ª CIET e incorporada também no SNA-1993 e em sua revisão, o SNA-2008 (ILO, 2000; UN, 2009, 1993).
} 


\section{B) PRODUÇÃO DAS FAMÍLIAS PARA O PRÓPRIO USO}

Compreende a produção de bens para o próprio consumo ou de serviços para o próprio uso. Inclui a produção de alimentos e pecuária ou de outros bens para consumo próprio, a própria construção ou a própria formação de capital fixo, o aluguel imputado e o serviço doméstico remunerado. Deve-se incluir também nesta categoria a produção de outros empreendimentos familiares que não sejam classificados como informais, como as atividades de aluguel efetivo e da agropecuária familiar voltada para o mercado. Segundo o Manual de ENO (OECD, 2002, p. 41):

For complete consistency this problem area should be defined as production performed by enterprises that are not classified as formal or informal, thereby including any other enterprise that falls outside the formal/informal distinction in addition to those producing for their own final use. (OECD, 2002, p. 41)

Com a inclusão destes outros empreendimentos familiares, esta área da ENO passa a se constituir no complemento ao setor informal do setor de produção famílias. $\mathrm{Na}$ apresentação dos resultados no item 4, tal categoria será designada como "outras unidades familiares".

\section{C) PRODUÇÃO ILEGAL}

Definida como atividades produtivas que geram bens e serviços proibidos pela lei. Inclui também atividades produtivas que são geralmente legais, mas que se tornam ilegais quando produzidas por agentes não autorizados. Exemplos comuns de atividades ilegais são a produção e o tráfico de drogas ilícitas, a prostituição (em países onde ela é proibida), a venda de bebidas alcoólicas aos menores de idade, ou a realização de algum serviço profissional sem o registro legal da profissão. Atos criminosos como sequestro, roubo ou furto não geram produtos ou valor adicionado na economia - não são atividades produtivas - e, por definição, são excluídos da fronteira de produção e, consequentemente, da ENO.

\section{D) PRODUÇÃO SUBDECLARADA}

Refere-se às atividades produtivas que não são declaradas, integralmente ou parcialmente, às autoridades públicas a fim de evitar o pagamento de impostos e contribui- 
ções; o cumprimento de normas legais relacionadas com o trabalho, segurança ou saúde; o cumprimento de procedimentos administrativos, tais como, questionários estatísticos ou registros administrativos. A produção subdeclarada pode ou não ser legal, do ponto de vista jurídico, e ocorrer em qualquer atividade econômica nos setores empresariais ou nas famílias.

\section{E) PRODUÇÃO SUBCOBERTA POR DEFICIÊNCIA DA ESTATÍSTICA}

Consiste em parte da produção que não é captada em razão das deficiências do sistema estatístico. Tais deficiências podem ocorrer porque novas empresas ainda não foram incluídas nos cadastros estatísticos e, por isto, não foram investigadas. Outra razão pode ser a não resposta dos questionários em razão do não recebimento pelo destinatário, por exemplo, por causa de uma falta de atualização no endereço cadastral.

A categorização acima objetiva esclarecer que os processos de produção não registrados oficialmente possuem naturezas distintas, sendo este conhecimento o primeiro passo para se viabilizarem propostas e métodos para a correta estimação da ENO no âmbito das contas nacionais. Cabe ressaltar que a categorização apresentada não exclui a possibilidade de sobreposição entre os tipos de produção integrantes da ENO. A seguir, alguns exemplos elucidam esta questão:

- Caso parte da receita de um estabelecimento que esteja presente em um cadastro oficial não seja declarada, esta produção encontrar-se-á subdeclarada, fazendo parte da ENO;

- A atividade do comércio ambulante de produtos legais, como por exemplo, a venda de frutas nas ruas, será classificada como ENO, na produção informal. Caso o comerciante não declare a totalidade de seus rendimentos à pesquisa domiciliar, sua atividade, além de informal, será também subdeclarada.

- Se um comerciante transacionar drogas ilícitas e, ainda assim, declarar os rendimentos de sua atividade na pesquisa domiciliar, será classificado como informal e ilegal;

- Se este mesmo comerciante ocultar parte de sua renda à pesquisa domiciliar, sua produção além de informal e ilegal será também subdeclarada;

- Pequenos agricultores podem declarar ao censo agropecuário sua produção de leite ou de ovos para o próprio consumo. Neste caso, embora classificada como produção das famílias para o próprio uso, esta será considerada parte da economia observada; 
- Caso os rendimentos de uma empregada doméstica estejam corretamente auferidos na pesquisa domiciliar, esta produção familiar será considerada parte da economia observada. Entretanto, se os rendimentos não forem completamente declarados, haverá um componente de subdeclaração, que fará parte da ENO.

As definições das cinco categorias que integram a ENO e os exemplos anteriores revelam que algumas destas atividades podem estar relacionadas também com a economia observada. Assim, em razão dos problemas e das dificuldades empíricas inerentes à questão, as recomendações internacionais não exigem que se distingam cada uma das cinco áreas, ou mesmo a ENO isoladamente. O que realmente é crucial ao SCN é que o espaço da economia observada e não observado sejam incorporados na fronteira de produção do sistema - o conceito de cobertura exaustiva - segundo as possibilidades de estimação ou imputação que cada país tenha a partir das estatísticas disponíveis e que sejam consideradas em seu conjunto como a produção total da economia $^{11}$.

Os métodos de estimação da ENO, apresentados no item 4, aliados às particularidades da arquitetura do SCN, que considera o cruzamento de informações da ótica da produção, da despesa e da renda, permitem que a ENO seja estimada e agregada ao SCN, ainda que seus componentes não sejam identificados separadamente.

\section{PRINCIPAIS MÉTODOS PARA A CONTABILIZAÇÃO DA ENO}

Como a ENO tem sua origem nas deficiências dos programas de compilação de dados básicos, a maneira ideal para sua incorporação é o fortalecimento do sistema estatístico a fim de que a economia observada seja tão exaustiva quanto possível, minimizando o fenômeno não observado. Entretanto, além de dispendiosos em termos de recursos financeiros, tais esforços devem ser contínuos ao longo do tempo e, ainda assim, os dados básicos poderão não alcançar toda a produção definida na fronteira do SCN. Haverá sempre algumas atividades produtivas que não serão diretamente observadas (OECD, 2002, par. 5.1, p. 69).

\footnotetext{
11 "As the techniques in the handbook make clear, a specific measure of the NOE is not important in itself. Attention focuses on ensuring that the measurement of total activity is complete or exhaustive" (UN, 2009, p. 471).
} 
Para sanar estas insuficiências dos programas de compilação com a finalidade de alcançar a exaustividade na cobertura do SCN, alguns métodos de pesquisa ou de estimação são indicados. Tais métodos podem variar consideravelmente entre os países.

Algumas fontes são bastante comuns para este fim tais como censos demográficos ou econômicos, estatísticas de empresas, pesquisas domiciliares, pesquisas de força de trabalho, registros administrativos de empregos, fiscais e tributários, cadastros de seguridade social, registros policiais e estatísticas de comércio exterior. As pesquisas de força de trabalho, as pesquisas de orçamento familiar e os registros fiscais são também amplamente aplicados para uma variedade de atividades em diversos países. Outras fontes são utilizadas para levantamentos específicos de determinadas atividades em poucos países, como, por exemplo, o contrabando de cigarros, a produção ilegal de bebidas, a produção e comercialização de drogas ilícitas ou a incidência da prostituição ${ }^{12}$.

Os principais métodos utilizados para se agregar a ENO ao SCN podem ser classificados em dois tipos: A) métodos baseados na compilação estatística; e B) métodos baseados em técnicas de modelagem. Por uma série de razões o uso dos primeiros é recomendado, enquanto a aplicação de métodos baseados em técnicas de modelagem é desaconselhada ${ }^{13}$.

Os métodos baseados na compilação estatística, por sua vez, se dividem em estimativas diretas, que consiste na busca da informação por levantamentos diretos como, por exemplo, pesquisas sobre produção informal, orçamento familiar ou uso do tempo; ou em estimativas indiretas. Estes últimos resultam de estimações indiretas que são combinadas com outras informações disponíveis no SCN. Em resumo, os principais métodos de captação indireta são ${ }^{14}$ :

\section{A) MÉTODOS BASEADOS NA OFERTA}

Baseia-se em dados sobre o fornecimento de insumos que são usados para a produção de bens e serviços. Estes podem ser, por exemplo, a quantidade de trabalhadores, matérias-primas, terra, estoque de capital fixo, entre outros. Por este método, a produção e o

\footnotetext{
12 Um exemplo pode ser encontrado em UNECE et al. (2008), que apresenta um resumo da experiência de mensuração da economia ilegal nas contas nacionais da Ucrânia.

13 Ver item (d) a seguir.

${ }^{14}$ Uma exposição completa dos métodos indiretos de estimação é apresentada no capítulo 5 do Manual da ENO (OECD, 2002), referência para esta síntese.
} 
valor adicionado são calculados a partir dos dados de entrada em associação aos coeficientes técnicos de produção (por exemplo, relação CI/VP, relação VA/VP, produtividade). O mais significativo método baseado na oferta é o que utiliza o fator trabalho como insumo, que pode ser aplicado em diversas atividades (OECD, 2002, p. 72). Grande parte das estimativas realizadas pelo SCN do Brasil utiliza esta técnica de mensuração.

\section{B) MÉTODOS BASEADOS NA DEMANDA}

Tem o propósito de estimar a produção utilizando indicadores quantitativos de usos de bens e serviços específicos que suficientemente indicam a produção, tais como: materiais de construção, despesas domésticas de consumo final, utilização de matérias-primas para o processamento de produtos agrícolas, exportação de produtos; ou ainda, dados administrativos que indicam a demanda por um produto específico, como o registro de veículos ou licenças de construção, entre outros.

\section{C) MÉTODOS BASEADOS NO FLUXO DE PRODUTOS}

Fundamentam-se no equilíbrio entre recursos e usos de grupos de produtos por meio de equações contábeis, que levam em conta a oferta e a demanda dos produtos. Registros de demanda superior à oferta de determinado produto indicam que houve uma falha na compilação dos dados básicos de produção por alguma razão (subdeclaração, subcobertura, não resposta), havendo espaço para a estimação de uma produção não observada. Estes métodos têm efetividade somente se os dados referentes aos usos dos produtos são confiáveis a tal ponto de superarem a qualidade dos dados de oferta, que seria, então, acrescida. Exemplos internacionalmente comuns de imputação por este método ocorrem na construção civil e na fabricação de produtos do fumo, quando as pesquisas de consumo registram demandas superiores às ofertas desses produtos.

\section{D) MÉTODOS BASEADOS EM TÉCNICAS DE MODELAGEM}

Consiste-se em modelos macroeconômicos que fornecem estimativas da ENO e são utilizados, em geral, por pesquisadores independentes dos órgãos de contas nacionais, ou até mesmo dos institutos oficiais de estatísticas. Os modelos mais comuns são os modelos monetários e os modelos de indicador global. Os primeiros assumem que a produção não contabilizada pode ser modelada por intermédio de uma função estatís- 
tica em termos de estoque ou fluxos de moeda, atribuindo-se a uma maior proporção de moeda em poder do público, em relação à quantidade de depósitos, um maior percentual de economia não observada. O segundo tipo pressupõe que a produção não contabilizada seja modelada em termos de uma única variável a qual se acredita altamente correlacionada com o PIB, como por exemplo, o consumo de energia elétrica.

Tais métodos produzem estimativas para a ENO como um todo ou somente para um componente, denominado de "economia subterrânea" (underground economy). Diversas críticas são direcionadas aos métodos deste tipo no Manual da ENO (OECD, 2002, p. 187). Dentre elas destacam-se: i) a falta de clareza em relação à definição do que se procura medir - o que exatamente significa economia subterrânea sem considerar o marco teórico do SCN ${ }^{15}$; ii) a alta dependência dos modelos a pressupostos excessivamente simplistas; iii) a obtenção de diferentes resultados sob as mesmas circunstâncias; e iv) a falta de plausibilidade de muitos destes resultados. As publicações especializadas (OCDE, 2002; UNECE, 2008; Eurostat, 2010; para citar alguns) alertam que tais métodos devem ser evitados e que o uso dos dados básicos, seja pela estimação direta ou indireta, é sempre preferível às técnicas de modelagem. Tais dados, oriundos de diferentes fontes de pesquisa ou de registros oficiais, encontram-se disponíveis aos compiladores e demais pesquisadores na maioria dos países, constituindo-se uma riqueza estatística que não deve ser preterida por modelos simplistas e imprecisos.

É importante ressaltar que não há um método único utilizado internacionalmente; vários métodos ou combinações de métodos são geralmente aplicados, dependendo das características e das possibilidades do sistema estatístico de cada país. Sendo assim, a comparação internacional dos resultados da ENO, por conta dos diferentes métodos utilizados, deve ser vista com ressalvas. Ademais, deve-se ter atenção também com a comparação em um mesmo país ao longo do tempo, pois, em certos países, a cobertura e a qualidade das fontes de dados melhora em conjunto com o desenvolvimento dos sistemas estatísticos, implicando que atividades que podem ter sido anteriormente consideradas como não observadas serão cada vez mais estimadas no processo regular de compilação das contas nacionais, diminuindo, portanto, o peso da ENO. Assim, as variações na parcela da ENO no PIB podem ser tanto uma indicação de mudanças na economia real ou podem ocorrer por causa da evolução das fontes estatísticas básicas, ou pela combinação em qualquer grau de ambos os fatores (UNECE, 2008, p. 8).

\footnotetext{
15 Para ilustrar esta falta de unidade em torno do conceito a ser mensurado nos modelos deste tipo, Van Eck (1987) apud OECD (2002, p. 141), apresenta quase 30 (!) diferentes termos utilizados por seus autores como sinônimos para underground economy: alternate, autonomous, black, clandestine, grey, hidden, invisible, occult, parallel, shadow, unofficial etc.
} 
O mais recente levantamento internacional (UNECE, 2008) sobre as práticas correntes em relação à mensuração da ENO revelou que países como Rússia, Lituânia e Romênia estão dentre os que possuem a maior contribuição da ENO nas estimativas divulgadas de PIB, superando os 15\%. Por outro lado, países como Suécia, Austrália, Holanda e EUA informaram que menos de $1,5 \%$ do PIB corresponde às estimações da ENO, conforme ilustra o Gráfico 1. Os valores apresentados no Gráfico 1 devem ser interpretados como a proporção do PIB resultante da estimação por métodos de compilação estatística indireta para se alcançar a completa cobertura das atividades produtivas no país.

\section{Gráfico 1 - Atividades não observadas incluídas no PIB de países selecionados (participação percentual - anos em torno de 2000)}

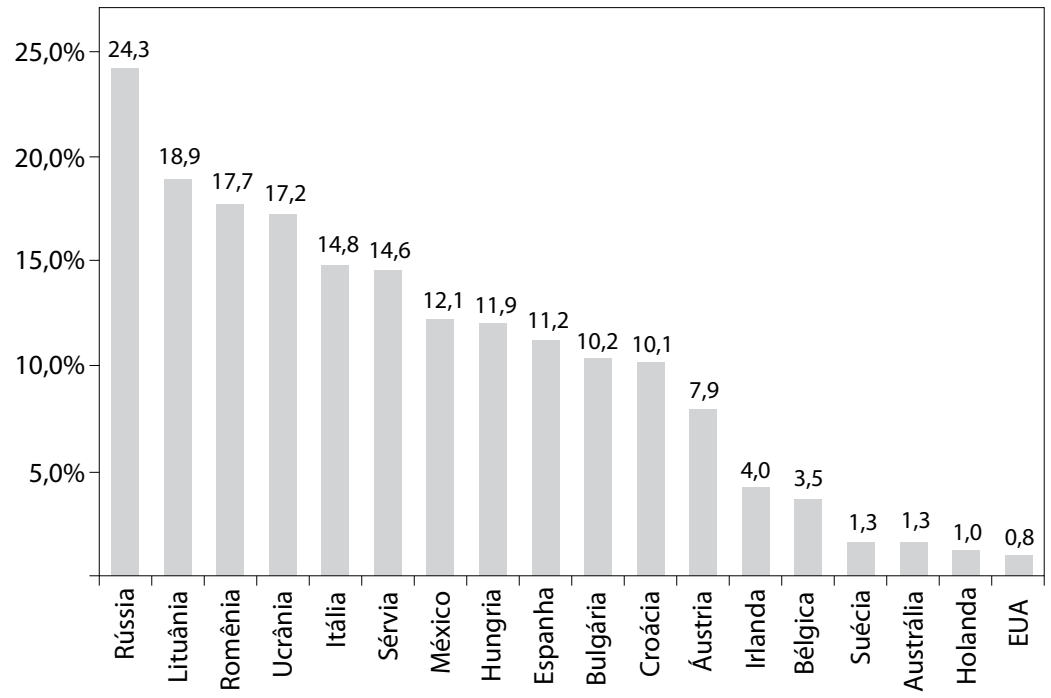

Fonte: Elaboração própria com base em UNECE (2008, p. 10).

\subsection{UMA ESTIMATIVA DA ENO PARA O BRASIL}

Assim como na maioria dos países, no Brasil também são aplicados diferentes métodos de estimação indireta para se alcançar a exaustividade do PIB, levando-se em conta os setores institucionais e os conjuntos de atividades econômicas que possuem características semelhantes em relação à função de produção. Na execução das contas nacionais brasileiras, os conjuntos de unidades que possuem características semelhantes ficam alocados em "modos de produção" próprios, definidos pelo sistema de compilação. 
Segundo IBGE (2006, p. 2), os modos de produção são utilizados nas contas de produção e de geração da renda, por atividade econômica, com uma tripla finalidade:

i) Diferenciar as funções de produção dentro de cada atividade econômica;

ii) Isolar as atividades informais e/ou subterrâneas;

iii) Facilitar a passagem das contas de atividades econômicas para as contas dos setores institucionais. (IBGE, 2006, p. 2, grifo dos autores).

Assim sendo, o segundo objetivo, destacado anteriormente, evidencia justamente a preocupação de separar as atividades econômicas que contribuem para a geração de valor adicionado e que são consideradas parte da ENO do país. Dos oito modos de produção existentes, numerados de 1 a 8 , dois deles são determinados para dar conta das estimativas de economia não observadas $-\mathrm{o} \mathrm{n}^{\circ} 6$, destinado às unidades produtivas familiares; e o $\mathrm{n}^{\circ} 7$, destinado à subdeclaração, que se refere "à produção não declarada pelas empresas" (IBGE, 2006, p. 2) ${ }^{16}$.

Segundo a metodologia das contas nacionais brasileiras, as estimativas de valor da produção e de valor adicionado para diversas atividades no setor famílias são obtidas utilizando os métodos baseados na oferta, considerando o fator trabalho como principal referência. Por sua vez, os resultados das atividades produtivas que têm origem subdeclarada ou subcoberta são estimados com base nos métodos baseados no fluxo de produtos ou na demanda (IBGE, 2008a, p. 52).

O principal método para as estimações de valor da produção e de valor adicionado no setor famílias considera como insumos a massa de rendimentos, por atividades econômicas, e como coeficiente técnico, a relação CI/VBP, também disponível para cada atividade. Os dados de emprego e rendimento por grupos de atividades econômicas tem como fonte a Pnad, e o coeficiente técnico de produção a Pesquisa de Economia Informal Urbana (Ecinf) ou o subgrupo de microempresas das pesquisas econômicas, também do IBGE. Com base nestes parâmetros estima-se a produção e o valor adicionado de um largo conjunto de atividades familiares ${ }^{17}$.

${ }^{16}$ Os demais modos de produção possuem uma estreita relação com os setores institucionais do SCN (empresas não financeiras, empresas financeiras, administração pública e instituições sem fins de lucro a serviço das famílias), sendo que alguns setores são alocados em mais de um modo por conta da natureza das fontes de dados. Por exemplo, se a fonte de informação da unidade for o estrato amostral da pesquisa econômica do IBGE, a alocação se realiza no modo de produção $n^{\circ} 1$; caso as informações da unidade sejam fornecidas pelo estrato amostral da pesquisa, o destino é o modo $n^{\circ} 3$. Para maiores informações, ver IBGE (2006).

17 Este procedimento é conhecido como "expansão da produção" no SCN. A nota metodológica do IBGE (2008a) traz os detalhes deste cálculo. 
O método de aproximação pelo fluxo de produtos e, em menor escala, o método baseado na demanda são também aplicados em diversas atividades durante o processo de compilação das contas nacionais brasileiras, mas no setor institucional empresas. Tais métodos visam contornar as deficiências de informação tanto de empresas não incluídas nos levantamentos estatísticos (subcobertura) quanto de lacunas relacionadas com a subdeclaração de receita por parte das unidades pesquisadas. Para cada grupo de produtos do SCN, quando os valores da demanda total (consumo intermediário e final, formação bruta de capital e exportação) superam os da oferta total (produção e importação), há uma indicação do tamanho do hiato de produção que deverá ser estimado e imputado nos balanços de recursos e usos. Cabe ressaltar a aplicação deste método nas contas nacionais brasileiras não possibilita a identificação das parcelas exclusivamente relacionadas com a subcobertura ou à subdeclaração, mas à soma de ambas.

Assim sendo, a magnitude da ENO brasileira será caracterizada, neste texto, como a soma dos resultados dos métodos de estimativas indiretas utilizados no SCN do Brasil: a expansão da produção para parte das atividades do setor famílias, que compreende principalmente a produção informal ${ }^{18}$; e o ajuste entre oferta e demanda que tem como causas a subdeclaração ou a subcobertura no setor empresarial. O cálculo apresentado refere-se ao período da nova série do SCN, que vai de 2000 a 2009. Convém destacar que estes resultados devem ser entendidos como a parcela do PIB, que é correntemente divulgado, oriunda de atividades não captadas diretamente pelo sistema estatístico e não que sejam em razão das atividades não mensuradas que deveriam então ser adicionados ao PIB.

Os resultados apresentados no Gráfico 2, estão, portanto, de acordo com marco teórico do SCN e com as recomendações internacionais para o cálculo da ENO. A soma das duas parcelas de estimação do $\mathrm{VAB}$ da economia brasileira pelos métodos descritos neste item alcançou 15,8\% do PIB, no ano base, e 11,6\%, no último ano da série. A redução da parcela da ENO de 4,2 p.p. ocorreu principalmente pela queda de 3,6 p.p. da estimativa da produção familiar, que continuamente perdeu peso desde o início da série. Enquanto o componente resultante da subdeclaração e da subcobertura nas empresas oscilou, em todo o período, entre 5,5\% (2002) e 4,2\% (2009), a estimativa para a atividade não observada familiar reduziu-se de 11,0\%, em 2000, para 7,4\%, em 2009.

\footnotetext{
${ }^{18}$ Embora a produção para o próprio uso seja um subgrupo da produção das famílias, seu peso na ENO brasileira é relativamente pequeno em razão da origem de seus resultados. Tanto para a agropecuária, quanto para o serviço doméstico remunerado, os resultados são observados pelo sistema estatístico regular por intermédio do censo agropecuário e da Pnad, respectivamente.
} 


\section{Gráfico 2 - Composição da ENO no Brasil obtida por métodos de estimação indireta segundo o setor institucional, 2000/2009 (em participação percentual em relação ao PIB)}

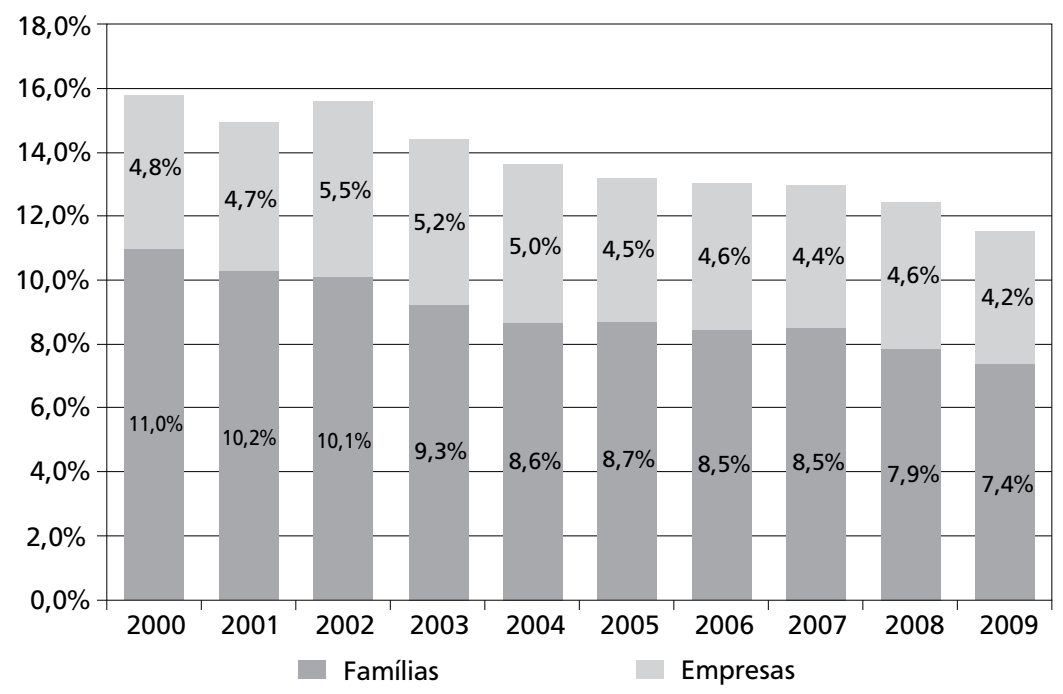

Fonte: Elaboração própria a partir da base de dados do SCN (IBGE, 2011).

A relativa estabilidade na estimativa da ENO em percentual do PIB encontrada pelos métodos baseados no fluxo de produtos e na demanda para o setor empresas é de certa forma esperada, pois ao longo dos dez anos de referência da série do SCN não houve modificações no sistema de coleta de informações estatísticas ou na compilação dos dados que justificassem sensíveis alterações em seus resultados. Tampouco este período foi marcado por instabilidades econômica, institucional ou jurídica que justificassem fortes variações nos percentuais de subdeclaração ou de não resposta pelas empresas ao sistema estatístico nacional. Ou seja, a manutenção da metodologia de obtenção dos dados e dos procedimentos nas fases seguintes, aliados a um ambiente econômico e jurídico estável, implicou uma limitada oscilação dos pesos da subcobertura e da subdeclaração no PIB brasileiro.

Com relação ao resultado encontrado para as atividades produtivas realizadas pelas famílias, obtido pelo método baseado na oferta, sua redução de peso pode ser atribuída às mudanças observadas na economia do país ao longo da década de 2000, tais como a trajetória ascendente assumida pelo emprego formal e a queda relativa da informalidade no mercado de trabalho brasileiro. De 2000 a 2009, enquanto as ocupações formais aumentaram em 50,7\%, as ocupações de trabalhadores sem carteira assinada cresceram $11 \%$ e as ocupações de autônomos somente $1,1 \%$, segundo as contas 
nacionais. Esta mudança na composição do emprego brasileiro refletiu-se na redução de peso da atividade produtiva do setor informal, e, consequentemente, da ENO obtida pelo método de estimação baseado na oferta. A relação entre a maior formalização do emprego e a redução da ENO se justifica, pois as ocupações de trabalhadores autônomos são características do setor informal, enquanto que as ocupações formais, em sua grande maioria, situam-se no setor formal, fazendo parte da economia observada ${ }^{19}$.

\section{Gráfico 3 - Posição relativa do Brasil segundo o peso da ENO no PIB: \\ Brasil (2000 e 2009) e demais países (anos em torno de 2000)}

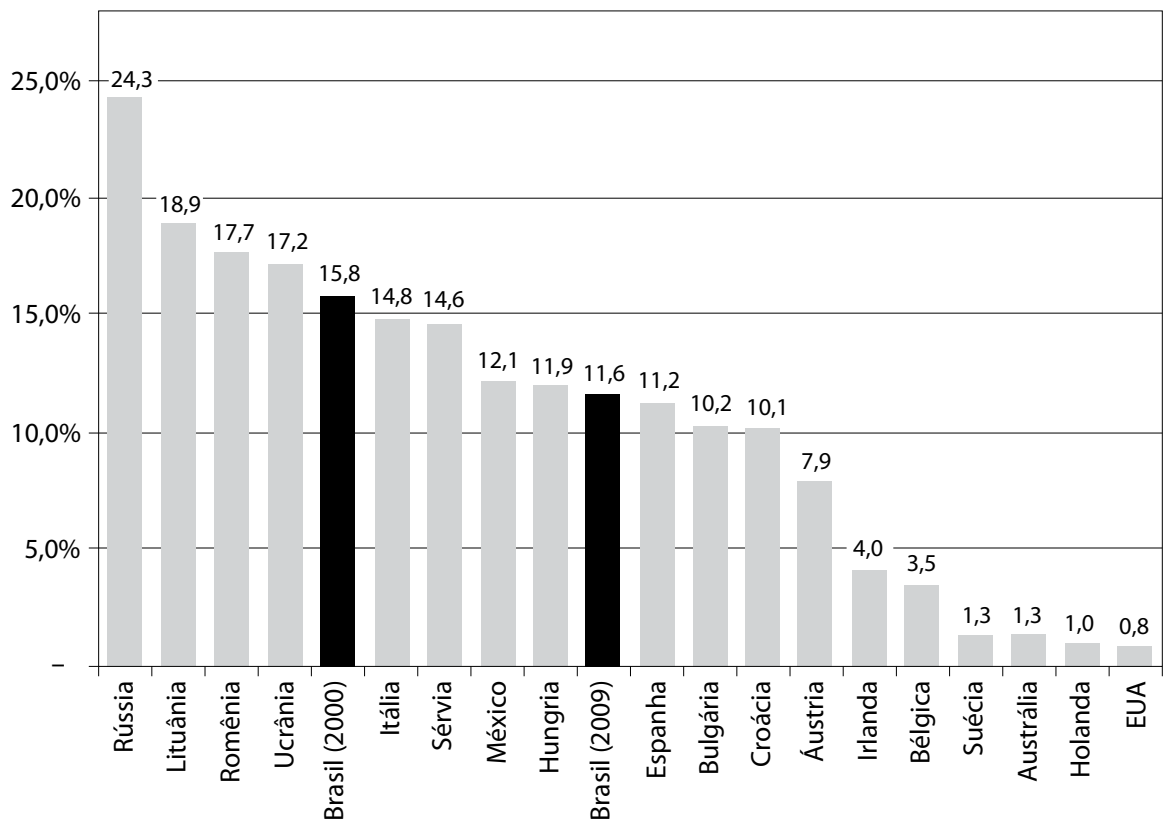

Fonte: Elaboração própria a partir da base de dados do SCN e de UNECE (2008).

Comparando o peso da ENO brasileira, no ano 2000, com os resultados dos dezoito países apresentados anteriormente, exibidos no Gráfico 3 a seguir, percebe-se que o Brasil situa-se na quinta posição dentre os países de maior taxa de PIB não observado, seguido por Itália (14,8\%) e Sérvia (14,6\%). Caso a comparação recaia sobre o resultado encontrado em 2009, o país passaria para a nona colocação, próximo ao

\footnotetext{
19 Para uma análise detalhada sobre os resultados de emprego por setor de produção e das possíveis causas
} para a redução do peso do setor informal na economia brasileira, ver Hallak (2013). 
México (12,1\%), Hungria (11,9\%) e Espanha (11,2\%). Os valores registrados pelo Brasil, tanto no início quanto no fim da série, se devem fundamentalmente às características da economia brasileira que, embora em trajetória descendente, concentra ainda um peso significativo na produção familiar e informal.

\section{CONSIDERAÇÕES FINAIS}

O tema da ENO tem sua origem no marco teórico das contas nacionais, uma vez que está atrelado à questão da necessidade de obtenção de uma cobertura econômica completa das atividades produtivas em seu âmbito. Apesar do contínuo investimento dos sistemas estatísticos nacionais para se coletar as informações da economia de maneira exaustiva, algumas atividades, por sua natureza, ou pela forma do método de investigação, escapam das estatísticas regulares. Assim, as fontes de dados que alimentam o sistema estatístico estão sujeitas, em maior ou menor grau, ao que ficou denominado na literatura como estatística subterrânea, que acarretam deficiências nos programas de compilação de dados básicos. Estas deficiências, que dão origem à ENO, estão comumente associadas à subcobertura de unidades produtivas, a não resposta por parte das unidades ou à subdeclaração deliberada por parte de empreendimentos que foram investigados pelas fontes de informação.

Qualquer atividade que esteja sob os limites da fronteira da produção deve ser contabilizada nos cálculos do SCN para efeitos de coerência interna, qualidade das estatísticas e, consequentemente, para sua maior utilidade qualquer que seja sua aplicação. As atividades que compõe a ENO estão contempladas na fronteira de produção e, por isso, devem ser incorporadas às contas nacionais para que sua cobertura seja exaustiva. O conteúdo da ENO compreende a produção informal e a produção para o próprio uso nas famílias, a produção ilegal, a produção subdeclarada e a produção subcoberta por deficiência da estatística.

Os principais métodos para se atingir a exaustividade da cobertura econômica são os métodos baseados na compilação estatística, que pode ser alcançada diretamente ou indiretamente. Os primeiros buscam, por meio de novas fontes de informação ou de melhorias em fontes já existentes, reduzir a parcela de ENO na economia total, ampliando a participação da economia observada. Os métodos apoiados na compilação estatística indireta resultam de estimações indiretas, baseadas na oferta, na demanda ou no fluxo de produtos específicos, combinadas com outras informações disponíveis, para estimar a ENO e agregá-la ao SCN. Os métodos derivados de técnicas de modelagem também produzem estimativas de economia subterrânea. Entretanto, em razão das falta de clareza conceitual do que se pretende mensurar, à excessiva sim- 
plicidade de seus pressupostos e à baixa qualidade de seus resultados, não são recomendados pelas publicações especializadas.

A estimativa da ENO para o Brasil, fundamentadas em estimações indiretas resultou em proporções do PIB de 15,8\%, em 2000, e 11,6\%, em 2009. A trajetória declinante desta proporção se deveu principalmente à queda da produção relativa das famílias na economia brasileira no período. Sem deixar de considerar as dificuldades de se realizar comparações internacionais em relação à ENO, tal resultado situa o Brasil entre os países de maior taxa de PIB não observado, fundamentalmente por causa das características da economia brasileira que concentra ainda um peso significativo na produção familiar e informal.

\section{REFERÊNCIAS}

BLADES, D.; ROBERTS, D. Measuring the non-observed economy. Statistics Brief, OECD, n. 5, nov., 2002.

EUROSTAT. Essential SNA: Building the basics. Luxembourg: Publications Office of the European Union, 2010.

HALLAK NETO, J. A distribuição funcional da renda e a economia não observada no âmbito do Sistema de Contas Nacionais do Brasil. Tese de Doutorado em Economia. Instituto de Economia, Universidade Federal do Rio de Janeiro, Rio de Janeiro, 2013.

INSTITUTO BRASILEIRO DE GEOGRAFIA E ESTATÍSTICA (IBGE). Sistema de Contas Nacionais - Brasil 2005-2009. Rio de Janeiro: IBGE, 2011.

INSTITUTO BRASILEIRO DE GEOGRAFIA E ESTATÍSTICA (IBGE). Sistema de Contas Nacionais - Brasil - referência 2000. Nota metodológica n. 23: Expansão da Produção (versão para informação e comentários). Rio de Janeiro: IBGE, 2008a. Disponível em: <http://www. ibge.gov.br/home/estatistica/economia/contasnacionais/2009/default_SCN.shtm.> Acesso em: 10 jul. 2012.

INSTITUTO BRASILEIRO DE GEOGRAFIA E ESTATÍSTICA (IBGE). Sistema de Contas Nacionais Brasil. Séries Relatórios Metodológicos, Vol. 24. Rio de Janeiro: IBGE, 2008b.

INSTITUTO BRASILEIRO DE GEOGRAFIA E ESTATÍSTICA (IBGE). Sistema de Contas Nacionais - Brasil - referência 2000. Nota metodológica n. 5: Modos de Produção (versão para informação e comentários). Rio de Janeiro: IBGE, 2006. Disponível em: <http://www.ibge.gov.br/ home/estatistica/economia/contasnacionais/2009/default_SCN.shtm> Acesso em: 10 jul. 2012.

INTERNATIONAL LABOUR ORGANISATION (ILO). "Resolution concerning statistics of employment in the informal sector, adopted by the Fifteenth International Conference of Labour Statisticians (January 1993)". In: ILO. Current International Recommendations on Labour Statistics. 2000 Edition. Geneva: International Labour Office, 2000.

MELO, H.; CONSIDERA, C.; DI SABBATO, A. Os afazeres domésticos contam. Texto para Discussão, UFF, n. 177, set. 2005. 
ORGANISATION FOR ECONOMIC CO-OPERATION AND DEVELOPMENT (OECD). $M e$ asuring the Non-observed Economy: a Handbook. Paris: OECD, 2002.

UKRAINE. Statistics Committee of Ukraine. Estimating the illegal economic activities in the national accounts. In: Conference of European Statisticians: measuring the non-observed economy in national accounts - illegal activities, 9, Geneva, 21-24 Apr. 2008. Disponível em: <http://www.unece.org/fileadmin/DAM/stats/documents/ece/ces/ge.20/2008/8.e.pdf>. Acesso em: 03 jul. 2012.

UNITED NATIONS (UN). System of National Accounts 1993 [SNA-93]. New York: UN, 1993. Disponível em: <http://unstats.un.org/unsd/nationalaccount/docs/1993sna.pdf>. Acesso em: 14 mar. 2012.

UNITED NATIONS (UN). System of National Accounts 2008 [SNA-2008]. New York: UN, 2009. Disponível em: <http://unstats.un.org/unsd/nationalaccount/docs/SNA2008.pdf>. Acesso em: 14 mar. 2012.

UNITED NATIONS ECONOMIC COMMISSION FOR EUROPE (UNECE). Inventory of national practices in estimating hidden and informal economic activities for national accounts. Geneva: UN, 1993. Disponível em: <http://www.unece.org/stats/publications/NOE1993. pdf>. Acesso em: 14 mar. 2012.

UNITED NATIONS ECONOMIC COMMISSION FOR EUROPE (UNECE). Draft Guidelines on Integrated Economic Statistics. United Nations Economic Commission for Europe. Prepared by the Friends of the Chair on Integrated Economic Statistics, Feb., 2012.

UNITED NATIONS ECONOMIC COMMISSION FOR EUROPE (UNECE). Non Observed Economy in National Accounts Survey of Country Practices. New York and Geneva: UN, 2008.

UNITED NATIONS ECONOMIC COMMISSION FOR EUROPE (UNECE). Non Observed Economy in National Accounts Survey of National Country Practices. Geneva: UN, 2003. 